\title{
Site Specificity in Femtosecond Laser Desorption of Neutral H Atoms from
} Graphite(0001)

Frigge, R.; Hoger, T.; Siemer, B.; Witte, H.; Silies, M.; Zacharias, H; Olsen, Thomas; Schiøtz, Jakob

Published in:

Physical Review Letters

Link to article, DOI:

10.1103/PhysRevLett.104.256102

Publication date:

2010

Document Version

Publisher's PDF, also known as Version of record

Link back to DTU Orbit

Citation (APA):

Frigge, R., Hoger, T., Siemer, B., Witte, H., Silies, M., Zacharias, H., Olsen, T., \& Schiøtz, J. (2010). Site Specificity in Femtosecond Laser Desorption of Neutral H Atoms from Graphite(0001). Physical Review Letters, 104(25), 256102. https://doi.org/10.1103/PhysRevLett.104.256102

\section{General rights}

Copyright and moral rights for the publications made accessible in the public portal are retained by the authors and/or other copyright owners and it is a condition of accessing publications that users recognise and abide by the legal requirements associated with these rights.

- Users may download and print one copy of any publication from the public portal for the purpose of private study or research.

- You may not further distribute the material or use it for any profit-making activity or commercial gain

- You may freely distribute the URL identifying the publication in the public portal 


\title{
Site Specificity in Femtosecond Laser Desorption of Neutral H Atoms from Graphite(0001)
}

\author{
R. Frigge, ${ }^{1, *}$ T. Hoger,${ }^{1}$ B. Siemer, ${ }^{1}$ H. Witte, ${ }^{1}$ M. Silies, ${ }^{1}$ H. Zacharias, ${ }^{1}$ T. Olsen, ${ }^{2}$ and J. Schiøtz ${ }^{2}$ \\ ${ }^{1}$ Physikalisches Institut and Center for Nanotechnology (CeNTech), Westfälische Wilhelms-Universität, 48149 Münster, Germany \\ ${ }^{2}$ Danish National Research Foundation's Center of Individual Nanoparticle Functionality (CINF), Department of Physics, \\ Technical University of Denmark, DK-2800 Kongens Lyngby, Denmark
}

(Received 1 April 2010; published 25 June 2010)

\begin{abstract}
Femtosecond laser excitation and density functional theory reveal site and vibrational state specificity in neutral atomic hydrogen desorption from graphite induced by multiple electronic transitions. Multimodal velocity distributions witness the participation of ortho and para pair states of chemisorbed hydrogen in the desorption process. Very slow velocities of 700 and $400 \mathrm{~ms}^{-1}$ for $\mathrm{H}$ and D atoms are associated with the desorption out of the highest vibrational state of a barrierless potential.
\end{abstract}

DOI: 10.1103/PhysRevLett.104.256102

PACS numbers: 68.43.Tj, 68.65.Pq, 82.20.Gk, 82.65.+r

The adsorption and desorption dynamics of hydrogen on graphite and graphene sheets has attracted considerable interest recently. In addition to the dramatic changes in the electronic properties of graphene, rendering it insulating $[1,2]$, atomic hydrogen chemisorbed on carbon scaffolds has been considered for hydrogen storage [3,4], a sink in plasma fusion devices [5], and a precursor for the formation of molecular hydrogen in interstellar molecular clouds [6]. Recent scanning tunneling microscopy revealed preferential sticking sites [7] in the case of preadsorbed $\mathrm{H}$ atoms and preferred sites for thermal reaction and desorption [8]. Also a tip-induced desorption of $\mathrm{H}$ atoms from graphene has recently been observed [9]. Theoretical calculations show that atomic hydrogen binds on top of carbon atoms with an energy of about $E_{b} \sim 0.7 \mathrm{eV}$. Thereby the $s p^{2}$ bound carbon scaffold is locally distorted and the now $s p^{3}$ binding $\mathrm{C}$ atoms pucker out of the graphite plane by about $0.3 \AA$. Because of this electronic change and atomic movement a barrier of about $0.25 \mathrm{eV}$ appears in the reaction pathway to chemisorption [10,11]. Co-adsorption of a second $\mathrm{H}$ atom in the vicinity of an already adsorbed one is preferred on the next neighbor ortho position and in the so-called para position located on the opposite site of the carbon hexagons. In these two positions the binding energy increases to about 1.9 to $2.1 \mathrm{eV}$, while in the nearest next neighbor position the binding energy remains unchanged [7,11]. In addition, the adsorption barrier is reduced in the ortho position, and even vanishes for adsorption in the para position. Further, energetic correlations over more extended regions of the carbon scaffold exist [12]. Correspondingly, evidence has been found that thermal molecular hydrogen formation occurs predominantly from such preferred pairs of atomic hydrogen [8]. Temperature programmed desorption (TPD) experiments show the formation of molecular hydrogen from chemisorbed atoms at temperatures in the range from 400 to $600 \mathrm{~K}$ and a first order desorption kinetics [13]. The double peak structure observed in TPD results from the recombination of $\mathrm{H}$ atoms out of these preferred pair sites on the graphite surface. Surface diffusion is not important, because the diffusion barrier on graphite is higher than $1 \mathrm{eV}$ thereby exceeding the binding energy of unpaired chemisorbed atoms and thus making diffusion unlikely [14].

Femtosecond laser excitation of graphite in the visible spectral range produces hot electrons which subsequently undergo various scattering processes, as electron-electron or electron-phonon scattering. Thereby a cloud of excited electrons is created which might scatter on adsorbates. Two-pulse time-correlated excitation experiments yield information about these electronic relaxations. Electronelectron scattering occurs on a time scale of a few tens of femtoseconds $[15,16]$ followed by phonon-mediated intraband cooling in 100 to $500 \mathrm{fs}$ and a corresponding population of the optical phonon mode in graphite [16-18]. This optical phonon shows a lifetime in the range of a few picoseconds and decays further into the phonon bath of the substrate [19].

In the present experiment a sample of highly oriented pyrolytic graphite with (0001) orientation is placed in an ultrahigh vacuum chamber at a base pressure in the low $10^{-10}$ mbar range. The sample is cleaned by cleaving the top layers just before placing it into the vacuum and then annealing it at temperatures of $1000 \mathrm{~K}$ for several minutes to remove any adsorbates. $\mathrm{H}$ and $\mathrm{D}$ atoms are produced by thermal dissociation in a tungsten capillary heated to about $2200 \mathrm{~K}$. The graphite sample at a distance of $80 \mathrm{~mm}$ from the capillary is exposed to the hydrogen flux at a chamber pressure of $5 \times 10^{-8} \mathrm{mbar}$ for about $20 \mathrm{~min}$ to ensure saturation, which corresponds to a coverage of $0.4 \mathrm{ML}$ [13]. After exposure to atomic hydrogen the surface is irradiated under $\theta=67.5^{\circ}$ by $p$-polarized pulses of a frequency doubled Ti:sapphire laser at a repetition rate of $10 \mathrm{~Hz}$. Pulses with a duration of about $35 \mathrm{fs}$ and energies up to $40 \mu \mathrm{J}$ are applied to an area of about $500 \times 780 \mu \mathrm{m}^{2}$. Using known electron-phonon coupling constants, it is estimated that at these laser intensities the distribution of hot electrons reaches peak temperatures of about $18000 \mathrm{~K}$. 


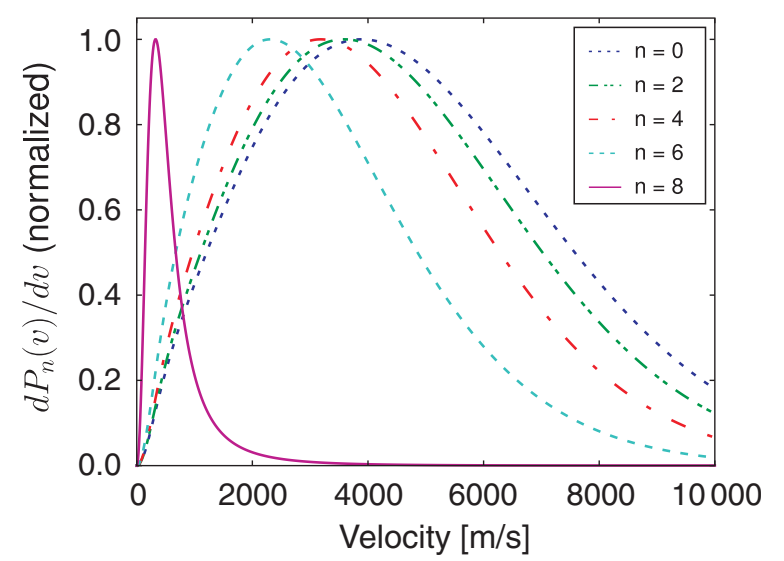

FIG. 1 (color online). Kinetic energy distribution for desorption out of five bound states for the para adsorption site, calculated from Eq. (2). The parameters are given in the text, and $\epsilon_{i}=\epsilon_{0}$. The probabilities have been normalized to fit the figure, in fact the maximum value of $P_{n}(v)$ increases by an order of magnitude for each $n$. The $n=8$ state thus has a much larger desorption probability than the rest.

Each laser pulse desorbs about $2.2 \times 10^{-5} \mathrm{ML}$ of the adsorbed hydrogen at dilute coverage, see below.

The desorbing hydrogen atoms are detected in the gas phase after a defined flight path and time delay by pulses of a tunable, frequency doubled dye laser. This laser operates at $\lambda=243 \mathrm{~nm}$, which excites the atoms via two-photon absorption in the $2 \mathrm{~s}^{2} S_{1 / 2} \leftarrow 1 \mathrm{~s}^{2} S_{1 / 2}$ transition. Absorption of a third photon ionizes only the electronically excited $\mathrm{H}$ atoms. The generated $\mathrm{H}^{+}\left(\mathrm{D}^{+}\right)$ions are detected by a time-of-flight mass spectrometer. Since the distance from the surface to the detection volume is known, the velocity of the desorbing atoms can be derived from the arrival time at the detection volume.

To calculate the atomic kinetic energy spectrum we employ scattering theory in conjunction with density functional theory (DFT). We consider an adsorbed atom which is described by an adiabatic potential $V_{0}(z)$ with desorption coordinate $z$ and a corresponding Hamiltonian $H_{0}$. The electronic system, which comprises both adsorbate and substrate, is described by a Newns-Anderson type Hamiltonian $H_{\mathrm{NA}}$ with a resonant electronic state $|a\rangle$ localized near the hydrogen atom and being unoccupied when the system is in the electronic ground state. When $|a\rangle$ becomes occupied the electronic structure changes and the Born-Oppenheimer potential of the adsorbate is altered to $V_{1}(z)$. The state $|a\rangle$ thus gives rise to a Hamiltonian term $H_{I}$ which couples the pure electronic term $H_{\mathrm{NA}}$ and the pure atomic term $H_{0}$. Even though the resonant state is short-lived, a transient occupation will perturb the system and may induce transitions between the bound states of $V_{0}(z)$ or from a bound to a dissociative state. The Hamiltonian describing the system is then

$$
H=H_{\mathrm{NA}}+H_{0}+H_{I}
$$

with

$$
\begin{gathered}
H_{\mathrm{NA}}=\varepsilon_{0} c_{a}^{\dagger} c_{a}+\sum_{q} \epsilon_{q} c_{q}^{\dagger} c_{q}+\sum_{q}\left(V_{a q} c_{a}^{\dagger} c_{q}+V_{a q}^{*} c_{q}^{\dagger} c_{a}\right), \\
H_{0}=\frac{1}{2} m \dot{z}^{2}+V_{0}(z), \\
H_{I}=\varepsilon_{a}(z) c_{a}^{\dagger} c_{a}-\varepsilon_{0} c_{a}^{\dagger} c_{a} .
\end{gathered}
$$

Here, $\epsilon_{a}(z)=V_{1}(z)-V_{0}(z)$ denotes the vertical potential energy difference, $c_{a}^{\dagger}$ and $c_{q}^{\dagger}$ are creation operators for the resonant state $|a\rangle$ and metallic states $|q\rangle$, respectively, and $\epsilon_{0}=\epsilon_{a}(0)$ [20,21]. Conceptually, the Hamiltonian describes an adsorbate with dynamics governed by $V_{0}(z)$ when the resonant state is unoccupied and $V_{1}(z)$ when the resonant state is occupied. To handle the model (1) we impose the wide band limit in which the resonance projected density of states is a Lorentzian with a full width at half maximum of $\Gamma$. Furthermore, we Taylor expand the interaction Hamiltonian to first order in the vicinity of the ground state minimum $(z=0)$ and get $H_{I}=-f_{a} z c_{a}^{\dagger} c_{a}$, where $f_{a}$ is the force felt by the adsorbate when the resonance is occupied. We can then use second order perturbation theory to calculate the differential probability $d P_{n}(k)$ that an incoming hot electron of energy $\epsilon_{i}$ will induce a transition in the adsorbate from a bound state $|n\rangle$ to a free state $|k\rangle$ [22]. The free states become plane waves asymptotically and can thus be converted into velocities. The result for the scattering probability is

$$
\begin{aligned}
d P_{n}(k)= & \frac{f_{a}^{2} \Gamma^{2}}{\left(E_{k}-E_{n}\right)^{2}}|\langle k|z| n\rangle|^{2} d k \mid \frac{1}{\varepsilon_{i}-\varepsilon_{0}+i \Gamma / 2} \\
& -\left.\frac{1}{\varepsilon_{i}-\varepsilon_{0}-\left(E_{k}-E_{n}\right)+i \Gamma / 2}\right|^{2},
\end{aligned}
$$

where $E_{n}$ and $E_{k}=\hbar^{2} k^{2} / 2 m$ are eigenenergies of bound and free states, respectively.

The parameters and potential energy surfaces in the model (1) have been calculated with the code GPAW $[23,24]$, which is a real-space density DFT code using the projector augmented wave method. The excited state potential energy surface was obtained with the linear expansion delta self-consistent field $(\Delta \mathrm{SCF})$ method $[21,25]$, where the resonant state is taken to be a localized antibonding $\mathrm{C}-\mathrm{H}$ orbital which is unoccupied without excitation. The resonance width $\Gamma$ is obtained from the KohnSham projected density of states. We will focus on the para and ortho dimer configurations which dominate at low coverage and are responsible for the two peaks observed in TPD spectra $[8,13]$. All calculations have been performed on a single graphene layer with a hydrogen dimer in a super cell containing 24 carbon atoms. We used a $4 \times$ $4 \mathrm{~K}$-point sampling, a grid spacing of $0.2 \AA$, and the PBE exchange-correlation functional [26]. The atomic adsorption potential for hydrogen in the para configuration has no barrier $[7,11]$ and can be approximated by a Morse poten- 
tial $V_{0}(z)=D\left(e^{-2 \alpha z}-2 e^{-\alpha z}\right)$ with $D=2.0 \mathrm{eV}$ and $\alpha=$ $3.5 \AA^{-1}$ yielding 9 bound states. In addition we find $\Gamma=$ $1.5 \mathrm{eV}, \epsilon_{0}=3.8 \mathrm{eV}$, and $f_{a}=0.27 \mathrm{eV} / \AA$. The eigenenergies $E_{n}$ and matrix elements $\langle n|z| k\rangle$ of the Morse potential are well known.

Figure 1 shows the kinetic energy spectrum according to Eq. (2) of desorbing $\mathrm{H}$ atoms associated with different bound states within the H-graphene potential for the para pair site. The positions of the peaks is roughly related to the widths of the bound state wave functions. The highest vibrational state is thus well separated from the others due to the fact that the potential is very wide close to the desorption energy. Since a desorption event is the result of multiple hot electrons sequentially exciting the adsorbate, the distribution of vibrational states is not in equilibrium with the graphite temperature at the time of desorption. To calculate the vibrational distribution resulting from scattering of multiple hot electrons, we use the model (1) to obtain the transition probability that a hot electron induces a vibrational transition $n \rightarrow m$ and obtain an expression similar to (2). Each hot electron thus changes the distribution induced by the previous hot electron and we can obtain the total yield by summing all $P_{n}(k)$ weighted by a distribution resulting from a given number of hot electrons. The desorption of atoms in the ortho configuration is treated in the same way except that $f_{a}=0.57 \mathrm{eV} / \AA$ and there is an adsorption barrier of $E_{B}=0.18 \mathrm{eV}$, which is included by accelerating desorbed atoms accordingly.

Experimentally, we measure the desorption yield and the velocity distribution of the desorbed $\mathrm{H}$ and $\mathrm{D}$ atoms. The atomic desorption yield shows for both $\mathrm{H}$ and $\mathrm{D}$ atoms a nonlinear dependence on the absorbed laser fluence with exponents of $n=(2.42 \pm 0.12)$ and $(1.85 \pm 0.11)$, respectively. This power law dependence hints to the fact that multiple electron scattering events are responsible for this DIMET desorption process [27]. Irradiating the surface at constant fluence without redosing the hydrogen coverage yields an effective desorption cross section. As is often the case in laser desorption experiments a fast initial decay followed by a significantly slower one is observed. At a fluence of $10 \mathrm{~mJ} / \mathrm{cm}^{2}$ we arrive at initial cross sections of $(2.5 \pm 1.0) \times 10^{-20} \mathrm{~cm}^{2}$ and $(4.9 \pm 1.0) \times 10^{-20} \mathrm{~cm}^{2}$ for $\mathrm{H}$ and $\mathrm{D}$ atoms, respectively, up to a total absorbed fluence of $5 \times 10^{19}$ photons $/ \mathrm{cm}^{2}$ or about 2500 pulses. After this initial irradiation coverages of about $0.13 \mathrm{ML}$ and $0.07 \mathrm{ML}$ of $\mathrm{H}$ and $\mathrm{D}$ atoms, respectively, remain on the graphite surface. For higher total fluences and thus lower remaining coverages lower cross sections of about $(5.6 \pm 0.2) \times$ $10^{-21} \mathrm{~cm}^{2}$ and $(4.1 \pm 0.1) \times 10^{-21} \mathrm{~cm}^{2}$ are, respectively, observed for $\mathrm{H}$ and $\mathrm{D}$ atoms. The initial desorption cross section at high surface coverage is thus significantly larger for the heavier isotopic species, while at more dilute coverages the cross sections are approximately equal for both isotopes. It should be kept in mind, however, that we measure in this way only a decrease in the hydrogen or

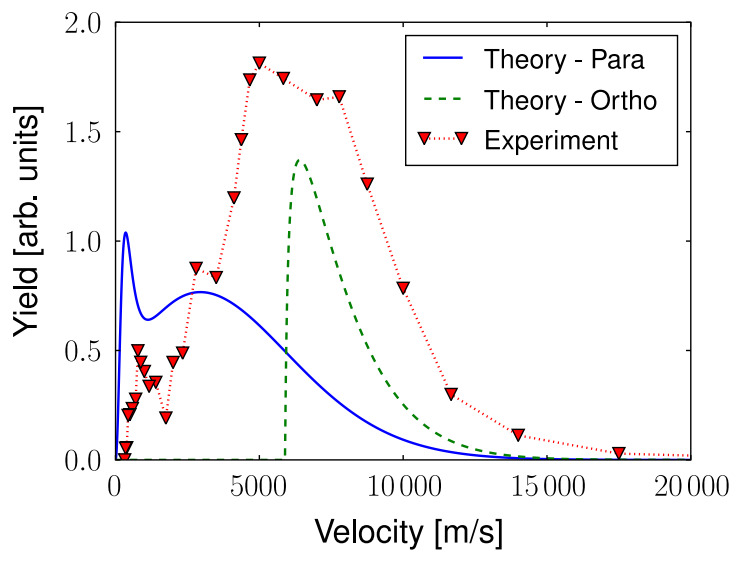

FIG. 2 (color online). Observed and calculated velocity distribution of $\mathrm{H}$ atoms from graphite. Triangles and dotted line denote the experimental data, the solid and dashed lines represent the theoretical results.

deuterium coverage of graphite. It may be speculated that the larger cross section is associated with the desorption of molecular hydrogen. Experiments to proof this conjecture by selective laser detection of $\mathrm{H}_{2}$ and $\mathrm{D}_{2}$ are currently in progress.

Velocity distributions have always been measured at dilute hydrogen coverages of the graphite surface of less than 0.1 ML by preirradiating the system accordingly. Figures 2 and 3 show the velocity distributions of $\mathrm{H}$ and $\mathrm{D}$ atoms, respectively, desorbing from graphite. Triangles and dotted lines denote the experimental data, and the solid and dashed lines represent results from the theoretical analysis. It is evident that multimodal velocity distributions are observed which can not be described by thermal distributions. For neutral hydrogen atoms a peak is observed around $v=700 \mathrm{~m} / \mathrm{s}$ and one around $v=5700 \mathrm{~m} / \mathrm{s}$, while for D atoms three peaks, at $v=400 \mathrm{~m} / \mathrm{s}$, $1600 \mathrm{~m} / \mathrm{s}$, and about $4400 \mathrm{~m} / \mathrm{s}$ can be discerned. Very intriguing are especially the peaks in the distributions at

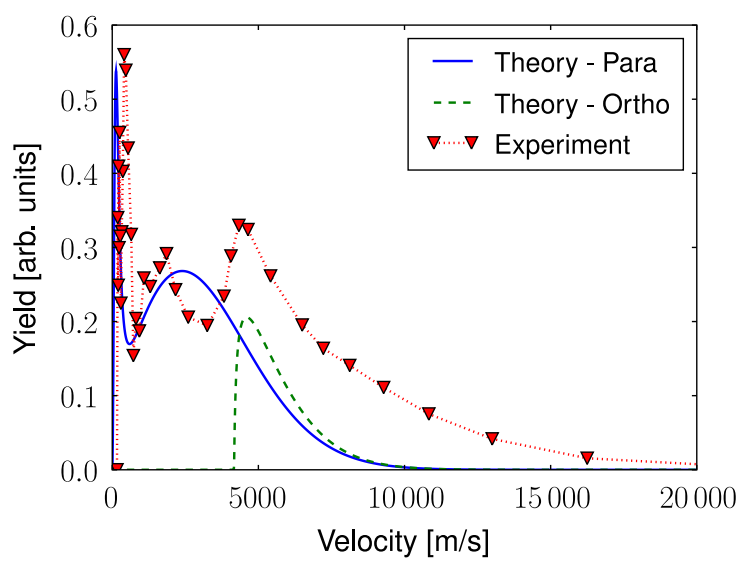

FIG. 3 (color online). Same as Fig. 2, but for D atoms. Note the distinct peak at very low velocities in both the experimental data and the theoretical results. 
very slow velocities. For assumed thermal distributions these peaks would correspond to kinetic energies of about 3 to $4 \mathrm{meV}$, or corresponding temperatures around 15 to $20 \mathrm{~K}$, which are not present in the system. On the other hand the peaks at high velocities correspond to average kinetic energies of about $\left\langle E_{\text {kin }}\right\rangle=224 \mathrm{meV}$ (H atoms) and $267 \mathrm{meV}$ (D atoms). It should further be noted that after velocity integration the observed yields for $\mathrm{H}$ and $\mathrm{D}$ atoms reveal that the total yield of desorbing $\mathrm{H}$ atoms is about 3 times higher than for D atoms. To recall, the initial deuterium coverage of graphite is much faster depleted than the hydrogen coverage, and at the dilute coverage both isotopic coverages are depleted with about the same effective cross section.

In Figs. 2 and 3 we compare the observed velocity distributions of hydrogen and deuterium with those calculated in the model. The solid lines in both figures indicate a good agreement between the experimental observations and first principals or model calculations. We have assumed that the distribution of vibrational states is the result of five hot electrons at resonance and that the fraction of para dimers is 10 times that of ortho dimers which is consistent with the observations of ref. [8]. While the choice of five initial hot electrons is rather arbitrary we have checked that the qualitative results do not depend crucially on this number. The positions of the peaks are independent of the initial distribution of vibrational states and changing the number of initial hot electrons only changes the relative magnitude of the peaks slightly. The two initial peaks at lower velocities are due to hydrogen atoms which originate from para states, while the high velocity peak reflects desorption from ortho states at which hydrogen atoms are accelerated by the barrier. In particular, one should note the low velocity peaks which result from desorption out of para dimers with atoms being in the highest vibrational states of the adsorption potential. The ortho configuration only shows a single peak, since the potential with a barrier does not have very wide vibrational states. Very fast atoms might origine from desorption out of monomer states which show an even higher barrier than the ortho states and, moreover, show a tighter binding to the graphene plane. According to the Franck-Condon principle this results in an excitation of the repulsive state at a higher energy.

The model used to calculate the desorption yield contains no fitted parameters, but one has to make an assumption on the distribution of hot electrons. Here we have made the simplest possible approximation, namely, all electrons being at the resonant energy. Increasing the number of contributing electrons would increase the population of high-lying vibrational states and thus change the ratio of the two para state peaks as well as increase the absolute value of the total calculated yield. In conclusion, we addressed the dynamics of photodesorption of neutral atomic hydrogen from the chemisorbed states on graphite. A DIMET process as suggested by the nonlinear yield dependence and the theoretical calculation is responsible for the desorption process. The observed velocity distributions can be associated with desorption out of ortho and para pair adsorption sites. Very low velocities result from desorption out of the highest vibrational state of the para pairs. We expect that these mechanisms play an important role also for the association reaction and in other systems.

*r.f@wwu.de

[1] J. O. Sofo, A. S. Chaudhari, and G. D. Barber, Phys. Rev. B 75, 153401 (2007).

[2] D. C. Elias et al., Science 323, 610 (2009).

[3] L. Schlapbach and A. Züttel, Nature (London) 414, 353 (2001).

[4] A. C. Dillon et al., Nature (London) 386, 377 (1997).

[5] C. H. Skinner and A. Haasz, Fusion Sci. Technol. 54, 891 (2008).

[6] D. Hollenbach and E. E. Salpeter, Astrophys. J. 163, 155 (1971).

[7] L. Hornekær et al., Phys. Rev. Lett. 97, 186102 (2006).

[8] L. Hornekær et al., Phys. Rev. Lett. 96, 156104 (2006).

[9] P. Sessi et al., Nano Lett. 9, 4343 (2009).

[10] X. Sha and B. Jackson, Surf. Sci. 496, 318 (2002).

[11] N. Rougeau, D. Teillet-Billy, and V. Sidis, Chem. Phys. Lett. 431, 135 (2006).

[12] Ž. Šljivančanin et al., J. Chem. Phys. 131, 084706 (2009).

[13] T. Zecho et al., J. Chem. Phys. 117, 8486 (2002).

[14] Y. Ferro et al., J. Chem. Phys. 120, 11882 (2004).

[15] S. Xu et al., Phys. Rev. Lett. 76, 483 (1996).

[16] M. Breusing, C. Ropers, and T. Elsaesser, Phys. Rev. Lett. 102, 086809 (2009).

[17] G. Moos et al., Phys. Rev. Lett. 87, 267402 (2001).

[18] H. Wang et al., Appl. Phys. Lett. 96, 081917 (2010).

[19] T. Kampfrath et al., Phys. Rev. Lett. 95, 187403 (2005).

[20] N. S. Wingreen, K. W. Jacobsen, and J. W. Wilkins, Phys. Rev. B 40, 11834 (1989).

[21] T. Olsen, J. Gavnholt, and J. Schiøtz, Phys. Rev. B 79, 035403 (2009).

[22] T. Olsen, Phys. Rev. B 79, 235414 (2009).

[23] The GPAW code is available as a part of the CAMPOS software: www.camd.dtu.dk/Software.

[24] J. J. Mortensen, L. B. Hansen, and K. W. Jacobsen, Phys. Rev. B 71, 035109 (2005).

[25] J. Gavnholt, T. Olsen, M. Engelund, and J. Schiøtz, Phys. Rev. B 78, 075441 (2008).

[26] J. P. Perdew, K. Burke, and M. Ernzerhof, Phys. Rev. Lett. 77, 3865 (1996).

[27] T. Olsen and J. Schiøtz, Phys. Rev. Lett. 103, 238301 (2009). 